\title{
Energy Harvesting Enabled Wireless Sensor Networks: Energy Model and Battery Dimensioning
}

\author{
Raul Gomez Cid-Fuentes, Albert Cabellos and Eduard Alarcon \\ NaNoNetworking Center in Catalunya (N3Cat) \\ Universitat Politècnica de Catalunya, 08034 Barcelona, Spain \\ \{rgomez, acabello \}@ac.upc.edu, eduard.alarcon@upc.edu
}

\begin{abstract}
Wireless Sensor Networks present a pending challenge for a complete deployability due to energy requirements. The low power density that these energy sources provide compared to the required energy for the communication process creates the necessity of temporal storage. Unfortunately, the random nature of the energy sources implies that the energy storage unit might not be able to guarantee the communication at all time, thus giving a certain loss probability, which is a function of the energy storage capacity. Typical solutions reduce this loss probability by over-dimensioning the battery, producing a very large overhead in size. In this paper, a scalable energy model is presented for the estimation of the loss probability. Accordingly, this energy model is applied in order to provide battery dimensioning guidelines. The results show that, by means of an accurate energy model, a certain loss probability can be achieved, while reducing up to 4 times the needed energy storage capacity.
\end{abstract}

\section{Categories and Subject Descriptors}

C.2.1 [Computer-Communication Networks]: Network Architecture and Design-Wireless Communication

\section{Keywords}

Wireless Sensor Networks, Energy Harvesting, Energy Model, Battery Dimensioning

\section{INTRODUCTION}

Advances in micro-electro-mechanical systems (MEMS) as a technology for new sensors and actuators, wireless communications and digital electronics have enabled the development of low-cost, low-power, small size, multi-application sensor nodes for Wireless Sensor Networks (WSN). The applications of this miniaturized sensors can be found in very diverse fields ranging from health monitoring [4] to environmental applications [7].

WSN targets the extension of the capabilities and applications of single nodes. This consists of the deployment of

Permission to make digital or hard copies of all or part of this work for personal or classroom use is granted without fee provided that copies are not made or distributed for profit or commercial advantage and that copies bear this notice and the full citation on the first page. To copy otherwise, to republish, to post on servers or to redistribute to lists, requires prior specific permission and/or a fee.

BODYNETS 2012 Oslo, Norway

Copyright 20XX ACM X-XXXXX-XX-X/XX/XX ...\$10.00. hundreds of coordinated sensors to provide high complexity applications in a distributed manner to cover large areas [2, $1]$.

One of the most active applications of WSN is on the development of Body Area Networks (BAN), that is the implantation of sensors in the human body to improve healthcare and the quality of life. Examples of applications for BAN are from remote health monitoring, sports training to secure authentication [3].

A major challenge in WSN is posed by the energy constraints of the nodes. In order to achieve the Self-Powered WSN paradigm, the limited energy storage of the nodes leads to the necessity of harvesting the available energy which can be found in the close environment of a sensor node. In general, the energy sources are presented in different natures, such as mechanical, thermal, solar, acoustic and electromagnetic energy [8]. Although this energy is usually considered as an unlimited source of energy, its low power density compared to the required energy to transmit information creates the necessity of temporal energy storage.

However, the bursty and random nature of the energy source, jointly with the communication process results in the fact that the energy storage unit might not be able to guarantee the communication at all time, thus forcing the sensor node to remain inactive during a certain fraction of time. This fraction of time, referred as the loss probability, strongly depends on the statistical properties of both harvesting and communication processes, as well as on the energy storage capacity.

Existing energy models $[9,6,5]$, usually target the optimization of some communication properties, as an example, the energy model in [6] provides the channel capacity in energy harvesting enabled nodes. However, up to now, energy models, are derived from the communication perspective and assume typical communication statistical distributions in order to model the energy harvesting process, which might not be a general assumption and, therefore, it might provide an inaccurate result. Due to the lack of an accurate energy model, and in particular, for this loss probability, it is common to over-dimension the energy storage, producing a large volume and weight overhead in the sensor node implementation.

In this context, we present design guidelines for battery dimensioning. These guidelines provide the minimum battery capacity required to achieve a certain probability of battery depletion, thus disabling the communication for a certain time. This is referred as loss probability. To do this, a scalable energy model is presented, which is able to capture different statistics for the harvesting source and it models 


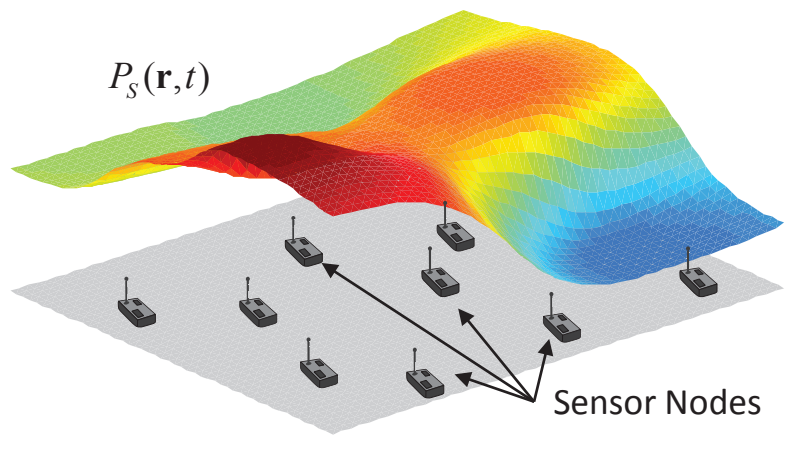

Figure 1: Qualitative representation of the energy field over the area.

the output energy as a communication process. Additionally, the harvesting energy fluctuations are separated into two dynamics; the smooth spatio-temporal variation which affects at the network level and the fast and uncorrelated variations which affects the statistics of the energy storage at the node level. These are referred as slow and fast dynamics respectively. This separation of dynamics enables the assumption that a single node is able to reach a steady state during a certain time and thus, enabling the characterization of the sensor node by means of its loss probability.

The rest of this paper is organized as follows. In Sec. 2, the energy harvesting model is described. In Sec. 3, the energy balance to achieve a perpetual operation is presented. In Sec. 4, the energy model is evaluated. In Sec. 5 design guidelines are discussed for the battery dimensioning. Finally, in Sec. 6 we conclude our work.

\section{ENERGY HARVESTING MODEL}

The energy available in the ambient is characterized by its ubiquitousness and perpetual character. This energy located near a single node is highly correlated in both time and space among neighboring nodes. However, there also exists a very fast variation due to the bursty nature of energy sources. These two effects enable the separation of dynamics to better understand and analyze the harvesting source. Therefore, we assume that the input power at a given time and position can be written as:

$$
P_{\text {harv }}(\mathbf{r}, t)=P_{S}(\mathbf{r}, t) \cdot p(\mathbf{r}, t)
$$

where $P_{\text {harv }}$ is the input harvesting power at a position $\mathbf{r}$ and time $t, P_{S}$ refers to the slow dynamics in power units and $p$ stands for the fast dynamics, which is described as a dimensionless stochastic process. In the following, we further relate the slow dynamics with the impact of energy harvesting at the network level, while the fast dynamics are related to the impact of harvesting at the node level. In any case, both dynamics are application dependent, so it is not possible to provide a closed-form, general expression. However, several properties of both dynamics are shown as follows and by assuming a certain nature of this energy source, this separation of dynamics can model the energy harvesting at the node and network level.

\subsection{Slow Dynamics}

The slow dynamics term is usually referred as the Energy field. This concept is shown in Fig. 1. Assuming that the nodes are deployed over a certain surface, the energy field provides the average input energy at each node. As shown, although the nodes are located in a discrete position, the Energy field is continuous in both, time and space, so it can easily capture nodes mobility or massively deployed networks. These slow dynamics have a strong impact at the network level. The figure shows the spatial distribution of the energy field at a given time.

The slow dynamics are usually generated by the addition of several independent energy sources, which might be located either inside or outside the network area. In this case, the resulting energy propagates from its source to the network area, and the power at a given point can be expressed as the addition of the convolution of these sources with the channel impulse response, if linear. This is:

$$
P_{S}(\mathbf{r}, t)=\sum_{i \in \mathcal{V}} V_{i}(\mathbf{r}, t) * g(\mathbf{r}, t)
$$

where $\mathcal{V}$ refers to the set of energy sources, $V_{i}$ stands for the generated power at the $i$-th source and $g$ stands for the impulse response of the channel.

Note that this model is useful as long as the number of energy sources is finite and considerably small, i.e. solar energy or vibrational energy in the close environment of a rotating engine. When the number of energy sources increases, statistical models to describe the energy field over the network area are more convenient, such as RF harvesting, where there are plenty of energy sources which are communicating in an uncoordinated manner. Therefore, there is also the need of a correlation coefficient which relates the variation of the energy along time and space. Being $i$ and $j$ two points in the space, the correlation coefficient is defined as:

$$
\rho_{i j}\left(t_{i}, t_{j}\right)=\frac{E\left[\left(P_{S, i}\left(t_{i}\right)-\mu_{i}\right)\left(P_{S, j}\left(t_{j}\right)-\mu_{j}\right)\right]}{\sigma_{i} \sigma_{j}}
$$

where $P_{S, i}$ and $P_{S, j}$ are the slow dynamics at a space points $i$ and $j$ at a time $t_{i}$ and $t_{j}$ respectively, $\mu_{i}$ and $\mu_{j}$ stand for the average input power, and $\sigma_{i}$ and $\sigma_{j}$ are standard deviations of the input power.

\subsection{Fast Dynamics}

At the node level, the energy is harvested from the close environment by means of energy scavengers. Then, the energy is power processed to guarantee the maximum power transfer and to adapt voltage levels. Afterwards, the input energy must be temporarily stored in a battery or capacitor to be later used in the node. Since the communication process is assumed to be the most energy consuming process in a node, the energy consumption given by the processing and sensing is not considered. Therefore, the energy stored is assumed to be later used during the communication process. This is referred as the power path. by:

The input energy at the node during a time $T$, is given

$$
e_{i n}=\int_{T} P_{h a r v}(\mathbf{r}, t) d t \approx P_{S} \int_{T} p(t) d t
$$

where the dependence with $\mathbf{r}$ is not considered since we consider a discrete location in space and $P_{S}$ is assumed to be constant due to different time dynamics. This input energy is modeled as a random variable which depends on the statistics of the random process $p(t)$. By calculating the variation, $\sigma_{e}^{2}$ of $e_{i n}$ in terms of its average value, $E\left[e_{i n}\right]$, it is obtained that $\sigma_{e}^{2} \propto E\left[e_{i n}\right]$. This is, although $p(t)$ can have 
any statistic, $e_{i n}$ is constrained to a set of statistics which achieve this condition between its mean and variance. As an example, the chi-square with $E\left[e_{i n}\right]$ degrees of freedom fulfills this condition.

Additionally, considering the condition between mean and variation and observing that $e_{i n}$ is proportional to the integration time, it is also true that the relation between deviation and mean tend to zero as long as the integration time increases. This is, large integration times tend to low-pass the variations of fast dynamics.

\section{ENERGY BALANCE}

During the node operation, the energy within the energy storage fluctuates due to the effect of the harvesting and communication processes. This energy level at the storage unit is referred as the energy state, $B$ and it is limited to the energy storage capacity $C$. This state will condition the operability of the sensor node.

In order to guarantee the operability of the node, the input energy, $e_{i n}$ must be higher than the output energy $e_{\text {out }}$, in average. This is, during a time $T$, it must be accomplished that:

$$
E\left[e_{i n}\right] \geq E\left[e_{\text {out }}\right]
$$

where $e_{\text {in }}$ and $e_{\text {out }}$ are the harvested energy and the communication energy expenditure during a time $T$. Note that the equality can only be achieved when the energy storage capacity tends to infinity. In harvesting-enabled, limited energy storage systems, the energy balance must operate near the equality as well to maximize the throughput. However, due to the randomness of both harvesting and communication processes, the instantaneous energy may not be balanced resulting in a certain loss probability $p_{\text {loss }}$.

\subsection{Communication Process}

The communication process consumes several orders of magnitude more instantaneous power than the energy harvesting power. Therefore, to keep the energy balance, the communication must be duty cycled in the sense that only for a very small portion of time the node is involved in the communication process, while most of time the node is sleeping. This fact enables the discretization of the communication energy expenditure. This is, the communication process shows an impulsive behavior. Commonly, this communication process is assumed to be Poisson distributed, having an exponentially distributed time between packets. The length of these packets can be either fixed or exponentially distributed.

\subsection{Energy State Characterization}

The energy state is conditioned by the following state equation:

$$
\frac{\partial B}{\partial t}=P_{\text {harv }}(t)-P_{\text {comm }}(t)
$$

where $B$ is the energy level at the battery, $P_{\text {harv }}$ is the harvesting power and $P_{\text {comm }}$ stands for the communication power. Additionally, the energy level at the battery is bounded by $0 \leq B \leq C$, where $C$ is the energy storage capacity

Since the communication process is impulsive and only the time when there is need for communication is required, the time can be discretized by the time between communication packets. Therefore, the energy variation during two communication packets is given by:

$$
\Delta B=e_{\text {in }}-e_{\text {out }}
$$

where $e_{i n}$ is the harvested energy during the time between two consecutive communication packets and $e_{\text {out }}$ is the packet energy. Since the time between packets is exponentially distributed, $e_{i n}$ is a function of the fast dynamics random process, $p(t)$, and the time between packets. Additionally, the equation is also bounded by $0 \leq B \leq C$

Since the battery capacity is the parameter of interest in battery dimensioning, this is also included in the above equation by dividing at both sides of the equation, this is:

$$
\Delta S=\frac{e_{\text {in }}}{C}-\frac{e_{\text {out }}}{C}
$$

where $S=B / C$, is the normalized energy state. $S$ can take any value from 0 to 1 . As shown, the normalized energy state only depends on the relation of energy with the storage capacity. This is, given a certain statistics for the harvesting process, the energy state is scalable.

\section{MODEL EVALUATION}

The loss probability is considered to be the main criterion for battery dimensioning. In this section we evaluate the loss probability in terms of the relation $C / e_{\text {out }}$ to provide design guidelines in the development of future autonomous sensor devices. $C / e_{\text {out }}$ is the relation between the energy storage capacity and the information packet energy (i.e. the total amount of information packets that can be transmitted without harvesting any energy).

To provide the following results, the equation (8) has been iterated in an event-based simulation. The parameters used in the simulation are $C=800 \mathrm{~J}, P_{S}=400 \mathrm{~mW}$, which are typical values in energy harvesting environments [8]. The required energy to transmit a packet $e_{\text {out }}$ is set as a design parameter and the time between packets $T$ is set such that $P_{S} T=e_{\text {out }}$. However, Finally, the loss probability has been defined as the probability of not having enough energy to transmit information when an information event occurs. This is $p_{\text {loss }}=\operatorname{Pr}\left\{S+e_{\text {in }}<e_{\text {out }}\right\}$.

\subsection{Impact of Different Statistics}

In Fig. 2(a), we show a comparison among the loss probability that different types of statistics at the energy model. In the figure, four situations are considered. Firstly, an exponentially distributed harvesting source is considered. This first energy source provides an input power which is exponentially distributed in amplitude. Additionally, a set of three chi-square distributed with an average $k=\{2.5,12.5, \infty\}$ degrees of freedom, where $k=\infty$ refers to constant input power, are considered. As shown, the effect of considering different statistics in the harvesting energy strongly affects the loss probability estimation. As an example, in order to achieve a loss probability of $10^{-3}$, a wrong estimation in the harvesting energy model would affect in an overdimensioning of up to 4 times in case of considering a Poisson distributed model for the harvesting process.

\subsection{Variations in the Input Energy}

In a real network, an error in the estimation of the harvesting rate may occur. Additionally, variations in the traffic patterns which may affect in an sudden increase or decrease of energy expenditure which may affect to the energy balance. This can have a dramatic impact in the loss proba- 


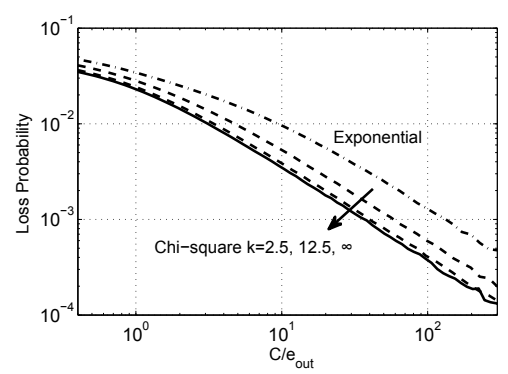

(a)

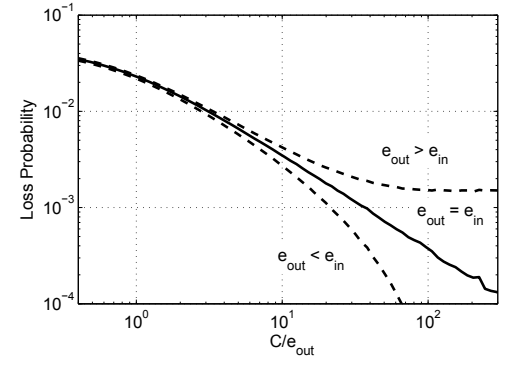

(b)

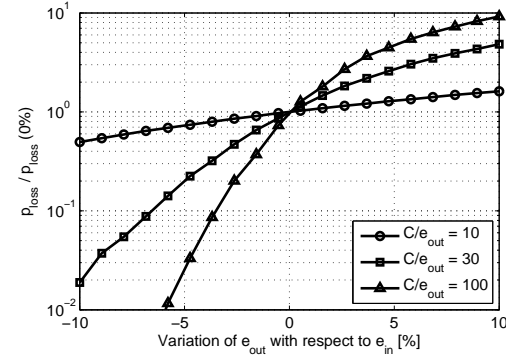

(c)

Figure 2: (a) Loss probability in terms of $C / e_{\text {out }}$ for exponential distribution (dash dotted line) and Chisquared (dashed and solid line), (b) impact on the loss probability of a small variation in the energy balance and (c) impact of the energy balance over the loss probability.

bility which must be considered in the future design before deployment.

Fig. 2(b) compares the energy balanced $\left(e_{\text {in }}=e_{\text {out }}\right)$ case to the situations where the input energy is either slightly above or slightly below the balanced situation. As shown, the loss probability is heavily affected by these two situations. Additionally, to better show this effect, Fig. 2(c) shows the impact of a variation in $e_{\text {out }}$ with respect to $e_{i n}$ in the loss probability. The impact in $p_{\text {loss }}$ is normalized by the value of $p_{\text {loss }}$ when $e_{\text {in }}=e_{\text {out }}$.

\section{BATTERY CAPACITY DESIGN GUIDE- LINES}

The statistical characterization of the node in terms of loss probability removes the effect of the fast dynamics and it enables the characterization of the loss probability in terms of the relation between the average input harvesting energy and the energy storage capacity. Therefore, the loss probability, $p_{l o s s}$, is given by a certain function

$$
p_{\text {loss }}=f\left(C / e_{i n}\right)
$$

where $f$ refers to the function which relates the loss probability with the relation between the energy storage capacity and average input harvesting energy (See Fig. 2(a)).

Therefore, having an average harvesting power $P_{S}$, and a certain communication rate, $R$ in packets per second units, the needed storage capacity to achieve a certain $p_{\text {loss }}$ is directly given by:

$$
C=\frac{P}{R} f^{-1}\left(p_{\text {loss }}\right) .
$$

In addition to this, in case of considering slow fadings or variations in traffic patterns, effect of energy unbalancing (See Fig. 2(c)) in the loss probability should also be included in (10) to guarantee the given loss probability.

\section{CONCLUSION}

Energy harvesting will have a great impact in the deployment of Wireless Sensor Networks (WSN) in almost every field of our society. For the time being, some solutions for energy harvesting have been already presented. However, the dimensioning of the energy storage is still a pending challenge, which is usually solved by over-dimensioning, hence being delimited in size and weight. In this paper, a novel energy model, which consists in the separation of dynamics, is presented. Additionally, the loss probability is obtained by using the presented energy model to provide design guidelines in the development of future wireless sensor netorks. The results show that the loss probability only depends in the relation between capacity and input energy, thus providing a fully scalable energy model. Moreover, it is shown the importance of accurately modeling the harvesting proces. An accurate model of the harvesting process can avoid the over-dimensioning, thus enabling a reduction in the energy storage capacity of up to 4 times.

\section{REFERENCES}

[1] I. F. Akyildiz and J. M. Jornet. Electromagnetic wireless nanosensor networks. Nano Communication Networks (Elsevier) Journal, 1(1):3-19, March 2010.

[2] I. F. Akyildiz, W. Su, Y. Sankarasubramaniam, and E. Cayirci. Wireless sensor networks: a survey. Elsevier Computer Networks, 38:393-422, 2002.

[3] M. Chen, S. Gonzalez, A. Vasilakos, H. Cao, and V. Leung. Body area networks: A survey. Mobile Networks and Applications, 16:171-193, 2011.

[4] J. M. Dubach, D. I. Harjes, and H. A. Clark. Fluorescent ion-selective nanosensors for intracellular analysis with improved lifetime and size. Nano Letters, 7(6):1827-2831, 2007.

[5] M. Gorlatova, A. Wallwater, and G. Zussman. Networking low-power energy harvesting devices: Measurements and algorithms. In Proc. of the IEEE INFOCOM, pages $1602-1610$, april 2011.

[6] R. Rajesh, V. Sharma, and P. Viswanath. Information capacity of energy harvesting sensor nodes. In Proc. of the IEEE International Symposium on Information Theory, pages 2363 -2367, 31 2011-aug. 52011.

[7] J. Riu, A. Maroto, and F. X. Rius. Nanosensors in environmental analysis. Talanta, 69(2):288 - 301, 2006.

[8] S. Sudevalayam and P. Kulkarni. Energy harvesting sensor nodes: Survey and implications. IEEE Communications Surveys Tutorials, 13(3):443 -461, 2011.

[9] J. Ventura and K. R. Chowdhury. Markov modeling of energy harvesting body sensor networks. In Proc. of IEEE PIMRC, Toronto, September 2011. 